Submitted to The Astrophysical Journal Letters

\title{
Is the Universe More Transparent to Gamma Rays than Previously Thought?
}

\author{
Floyd W. Stecker \\ Astrophysics Science Division, NASA Goddard Space Flight Center \\ Greenbelt, MD 207r71, U.S.A. \\ Floyd.W.Stecker@nasa.gov \\ Sean T. Scully \\ Department of Physics, \\ James Madison University, Harrisonburg, VA 2280\%, U.S.A. \\ scullyst@jmu.edu
}

\begin{abstract}
The MAGIC collaboration has recently reported the detection of the strong $\gamma$-ray blazar 3C279 during a 1-2 day flare. They have used their spectral observations to draw conclusions regarding upper limits on the opacity of the Universe to high energy $\gamma$-rays and, by implication, upper limits on the extragalactic mid-infrared background radiation. In this paper we examine the effect of $\gamma$-ray absorption by the extragalactic infrared radiation on intrinsic spectra for this blazar and compare our results with the observational data on $3 \mathrm{C} 279$. We find agreement with our previous results, contrary to the recent assertion of the MAGIC group that the Universe is more transparent to $\gamma$-rays than our calculations indicate. Our analysis indicates that in the energy range between $\sim 80$ and $\sim 500 \mathrm{GeV}, 3 \mathrm{C} 279$ has a best-fit intrinsic spectrum with a spectral index $\sim$ 1.78 using our fast evolution model and $\sim 2.19$ using our baseline model. However, we also find that spectral indices in the range of 1.0 to 3.0 are almost as equally acceptable as the best fit spectral indices. Assuming the same intrinsic spectral index for this flare as for the 1991 flare from 3C279 observed by EGRET, viz., 2.02, which lies between our best fit indeces, we estimate that the MAGIC flare was $\sim 3$ times brighter than the EGRET flare observed 15 years earlier.
\end{abstract}

Subject headings: gamma-rays: theory, galaxies ; 3C279: cosmology: diffuse radiation

\section{INTRODUCTION}

It has long been recognized that by studying the spectra of strong extragalactic $\gamma$-ray sources one can obtain information about the density and energy spectra of intergalactic photon fields. The 
luminous blazar 3C279 was discovered by the EGRET detector aboard the Compton Gamma Ray Observatory to be a strong flaring $\gamma$-ray source (Hartman et al. 1992). Shortly after this discovery, (Stecker, de Jager \& Salamon 1992) (hereafter SDS) proposed that the study of the TeV spectra of such sources could be used to probe the intergalactic infrared radiation.

SDS proposed to look for the energy dependent features indicating the mutual annihilation of very high energy $\gamma$-rays and low energy photons of galactic origin via the process of electronpositron pair production $\gamma+\gamma \rightarrow e^{+}+e^{-}$. The cross section for this process is exactly determined; it can be calculated using quantum electrodynamics (Breit \& Wheeler 1934). Thus, in principle, if one knows the emission spectrum of an extragalactic source at a given redshift, one can determine the column density of photons between the source and the Earth as a function of redshift.

Since the EGRET discovery of 3C279, the infrared background at wavelengths not totally dominated by galactic or zodiacal emission has been measured by the Cosmic Background Explorer (COBE). In addition, there have been extensive observations of IR emission from galaxies themselves, whose total emission is thought to make up the cosmic IR background (see review by Hauser \& Dwek 2001). The latest extensive observations have been made by the Spitzer satellite. It is thus appropriate to use a synoptic approach combining the very high energy $\gamma$-ray observations with the extragalactic IR observations, in order to best explore both the $\gamma$-ray emission from blazars and the diffuse extragalactic IR radiation. Using this approach, one must take account of both the high energy $\gamma$-ray observations and the data from the many galaxy observations presently available.

The MAGIC collaboration has recently observed the spectrum of the blazar 3C279 during a flare which occurred on 22-23 February 2006 (Albert et al. 2008). The highly luminous blazar 3C279 lies at a redshift of 0.536 (Marzioni et al.1996). To date, it is the most distant $\gamma$-ray source observed in the sub-TeV energy range. Thus, as noted by SDS, this source is potentially highly significant for probing the intergalactic background radiation.

Albert et al. (2008) used their observational data to draw conclusions regarding the maximum opacity of the Universe to $\gamma$-rays in the sub-TeV energy range. Their conclusions regarding the extragalactic background radiation would appear to disfavor the results of the extensive semiempirical calculations of the extragalactic IR background spectrum given by Stecker, Malkan \& Scully $(2006,2007)$ (hereafter SMS). In particular, the limit shown in their Figure 2 appears to be inconsistent with one of the SMS models that was based primarily on galaxy studies by the Spitzer infrared satellite telescope.

In this paper, we will reexamine both the analysis assumptions and the conclusions presented in the paper of Albert et al. Using a different analysis technique that we show to be superior to that of Albert et al. , we find that the observations of 3C279 are fully consistent with both diffuse IR background models obtained by SMS. We then discuss the implications of these results regarding both the intrinsic energy spectrum and luminosity of the $3 \mathrm{C} 279$ flare and the opacity of the Universe to $\gamma$-rays. 


\section{The diffuse extragalactic IR background}

Various calculations of the extragalactic IR background have been made (Stecker, Puget \& Fazio 1977; Malkan \& Stecker (1998, 2001); Totani \& Takeuchi (2002); Kneiske et al. (2004); Primack et al. (2005); SMS). Of these models, the most empirically based are those of Malkan \& Stecker (1998, 2001), Totani \& Takeuchi (2002) and SMS. Since the largest uncertainty in these calculations arises from the uncertainty in the temporal evolution of the star formation rate in galaxies, SMS assumed two different evolution models, viz., a "baseline" model and a "fast evolution" model. These models produced similar wavelength dependences for the spectral energy distribution of the extragalactic IR background, but gave a difference of roughly $30-40 \%$ in overall intensity.

The empirically based calculations mentioned above include the observationally based contributions of warm dust and emission bands from polycyclic aromatic hydrocarbon (PAH) molecules and silicates, which have been observed to contribute significantly to galaxy emission in the mid-IR (e.g., Lagache et al. 2004). These components of galactic IR emission have the effect of partially filling in the "valley" in the mid-IR spectral energy distribution between the peak from starlight emission and that from cold dust emission. The model of Primack et al. (2005), which was based on strictly theoretical galaxy spectra, does not take the warm dust, PAH, and silicate emission components of mid-IR galaxy spectra into account and therefore exhibits a steep mid-IR valley that is in direct conflict with solid lower limits obtained from galaxy counts obtained from observations of galaxies at mid-IR wavelengths (Altieri et al. 1999; (Elbaz et al2002). This is clearly shown in Figure 2 of the supplemental online material of Albert et al. (2008). However, since Albert et al. (2008) considered it to be a "lower limit" model, we will discuss the Primack et al. model in our analysis.

\section{The observed spectrum of 3C279 and its derived intrinsic spectrum}

According to the MAGIC analysis, the 2006 flare on 3C279 had an observed spectral index of $4.11 \pm 0.68$ in the energy range between $\sim 80$ and $\sim 500 \mathrm{GeV}$. In their analysis, the MAGIC group chose to multiply their data points by $e^{\tau\left(E_{\gamma}\right)}$, where the optical depth, $\left.\tau\left(E_{\gamma}\right)\right)$ is chosen by using the results of various optical depth calculations. They then fit simple power-law spectra to the resulting fluxes. Using estimated optical depths for only the fast evolution model of SMS, they

gave a best-fit power-law spectral index for the intrinsic source spectrum of $\Gamma_{s}$ of $0.49 \pm 1.19$ for the which they imply is ruled out by invoking the questionable criterion $\Gamma_{s} \geq 1.5$ (However, see, e.g., (Katarzyńsky et al. 2006; Stecker, Baring \& Summerlin 2007; Resmi \& Bhattacharya 2008).

In this paper, we will adopt a different method for analyzing the intrinsic spectrum of $3 \mathrm{C} 279$, based on the one we first used for the analysis of the H.E.S.S. observations of the source 1ES0229 +200 (Stecker \& Scully 2006). The method is superior to the approach used by the MAGIC collaboration where one chooses to force a power-law fit to the implied deabsorbed data points. 
Instead we assume an intrinsic power-law spectrum emitted by the source over the limited observed energy range that covers less than a decade in energy. In order to compare with the observations, we multiply this power law by an absorption factor $e^{-\tau\left(E_{\gamma}, z=0.536\right)}$, where the optical depth, $\tau$, is calculated for a redshift $\mathrm{z}=0.536$. We then employ a nonlinear least squares fit of our two parameter model to the observational data.

Our method has several advantages over that chosen by the MAGIC collaboration. To begin with, the redshift of the source and the energy range of the observations implies that even the counts in the lowest MAGIC energy bin have been affected by at least some amount of intergalactic absorption. As a result, multiplying the data by $e^{\tau}$ and then fitting an arbitrary power-law as done by Albert et al. (2008) does not allow a proper fit to the normalization of the spectrum. Both the true blazar luminosity and the true form of the intrinsic spectrum are masked. Furthermore, the actual intrinsic spectrum cannot be assumed to have a power-law form after multiplication by an exponential that is nonlinear in energy at this particular redshift (see, however, Stecker \& Scully 2006). Also, in order to properly account for the effect of the optical depth, one should directly include it in the unfolding method used to produce the fluxes from the raw photon counts. Since the MAGIC data points represent a mean for an energy bin, it is not sufficient to simply multiply that mean point by $e^{\tau}$. The function $e^{\tau\left(E_{\gamma}\right)}$ is a rapidly changing function of energy. This fact must be taken into account when computing both the spectrum and error bars. The implied weighting towards a lower energy within a bin indicates that the appropriate value for $\tau(E)$ should be lower than that assumed for the mean energy in the bin. Thus, the values for $\tau$ used in the MAGIC analysis are too high for all of the EBL models they used.

\section{Analysis}

We begin our analysis program by calculating the optical depths in the energy range of the observations for $\mathrm{z}=0.536$ for both the SMS fast evolution model and baseline model. For comparison, we also consider the optical depths taken from Primack et al. (2005) and two of the models of Kneiske et al. (2004), viz., their best-fit model and their low-IR model. We find that all five of the optical depth models considered are well fit by third order polynomials in the energy range of interest of the form shown in equation (1).

$$
\log \tau=a_{1} \log ^{3} E_{\gamma}+a_{2} \log ^{2} E_{\gamma}+a_{3} \log E_{\gamma}+a_{4}
$$

Table 1 summarizes the fit parameters to the five models considered here. Figure 1 shows the excellent agreement of the third order polynomial fits to optical depths for the five models.

Because of the nonlinear nature of the energy dependence of the optical depth, we do not expect that the observed spectrum will have a power-law form. Thus, we fit to spectra that are assumed to be power-law intrinsic source spectra $\left(K E_{\gamma}{ }^{-\Gamma}\right)$ multiplied by $e^{-\tau}$ as prescribed by equation (1) using the various optical depth models indicated in Table 1 to take account of intergalactic 
absorption. We then employ the Levenberg-Marquardt nonlinear least squares method to fit the resulting nonlinear spectra to the observed data. We choose to fit to the data using only statistical errors.

We first do a $\chi^{2}$ a fit with two free parameters, viz., the normalization coefficient, $K$ and the intrinsic spectral index of the source $\Gamma_{s}$. The best-fit spectral curves for the various EBL models considered are shown in Figure 2. The corresponding best-fit values $K$ and $\Gamma_{s}$ are given in Table 2. In this table, in the first column, the $K$ values are given for $E$ in $\mathrm{GeV}$. The second column gives the best-fit spectral indeces for all of the models. The third column gives the $\chi^{2}$ values for the two-parameter best fit. It can be seen from this column that although the best-fit spectral indeces are significantly different for the various EBL models, the best fit $\chi^{2}$ values are comparable for all of the models.

The Levenberg-Marquardt method yields substantially different best-fit spectral indices for our modeled optical depths and those of Kneiske et al. (2004) and Primack et al. (2005). However, these fits are not particularly unique in their goodness of fit. We performed a $\chi^{2}$ analysis on all of the models, allowing both $\Gamma_{s}$ and $K$ to be free parameters in the fit. As can be seen from Table 2, the form of the intrinsic spectrum of the 3C279 flare is undetermined by the five data bins obtained by the MAGIC collaboration, even when one only considers the large statistical errors involved and neglects the significant systematic errors. This is because the lower energy points with the smaller error bars are weighted more highly than the highest energy point.

Since the minimum $\chi^{2}$ is almost independent of choice of spectral index, the two-parameter fitting routine mainly tries to move the curve up and down (i.e. adjusts the normalization) to best fit the observational data. This feature is lacking in the MAGIC analysis of the spectrum of 3C279 since they have factored in the absorption effect prior to making their fits.

To further illustrate this point of ambiguity in the spectral index of the source, Figure 3 shows the best fit obtained for the SMS baseline model to the observational data for the range of spectral indices indicated. Spectra with the higher values of $\Gamma_{s}$ provide a good fit to the three lower energy

Table 1. Parameters Used in Equation (1) for Opacity Models

\begin{tabular}{lllll}
\hline \hline \multicolumn{1}{c}{ Model } & \multicolumn{1}{c}{$a_{1}$} & $a_{2}$ & \multicolumn{1}{c}{$a_{3}$} & \multicolumn{1}{c}{$a_{4}$} \\
\hline Fast Evolution & 0.0999257 & -1.26786 & 5.36642 & -6.21713 \\
Baseline & 0.103179 & -1.28947 & 5.41409 & -6.36993 \\
Kneiske Best Fit & -0.477755 & 2.84965 & -3.77753 & -0.353419 \\
Kneiske Low IR & -0.334462 & 1.75885 & -1.19612 & -2.30134 \\
Primack & -0.549224 & 3.43815 & -5.63807 & 1.57098 \\
\hline
\end{tabular}




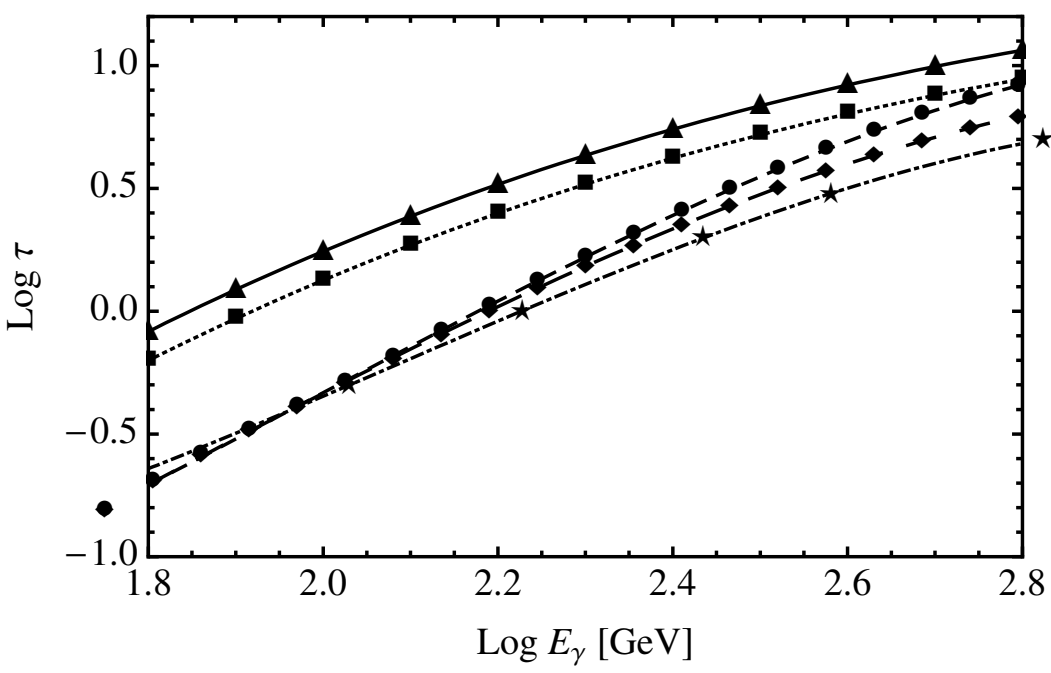

Fig. 1.- Polynomial fits to the five optical depth models discussed in the text. The solid line and dotted line are fits to the SMS fast evolution (triangles) and baseline (filled boxes) models respectively. The short-dashed and long-dashed lines are polynomial fits to the best-fit Kneiske et al. model (filled circles) and low-IR Kneiske et al. model (diamonds) respectively. The dot-dashed line is the polynomial fit to the Primack et al. optical depth model (stars).

data points which have the smallest error bars. However, they miss the higher energy data points. Those with lower values of $\Gamma_{s}$ miss the lower energy data point but pass through the four higher energy data points. all of these fits have almost identical reduced $\chi^{2}$ values of $\sim 1.1$. Choosing a range of $\Gamma_{s}$ between 1.5 and 3 as shown in Figure 3, and varying the normalization constant, $K$ to find the best fit in each case, it is found that all of these spectral fits have almost identical reduced $\chi^{2}$ values of $\sim 1.1$. As a result, all of them are equally probable. Other EBL models exhibit the same degeneracy in $\chi^{2}$ over a range of spectral indeces.

\section{Hypothetical Consideration of the Luminosity of the 3C279 Flare Observed by MAGIC}

As discussed above, our statistical analysis shows that the spectral index of the flare is not well determined by the MAGIC data. Given this uncertainty, we can conditionally compare the luminosity of the MAGIC flare with that of an earlier flare observed by EGRET by assuming the same intrinsic spectral index as the EGRET flare. The 1991 EGRET flare had an observed spectral index of $\Gamma_{s}=2.02$ at energies between $50 \mathrm{MeV}$ and $10 \mathrm{GeV}$ (Hartman et al. 1992) where EBL absorption is negligible.

We note that this choice of spectral index gives good $\chi^{2}$ fits for all the EBL models considered 


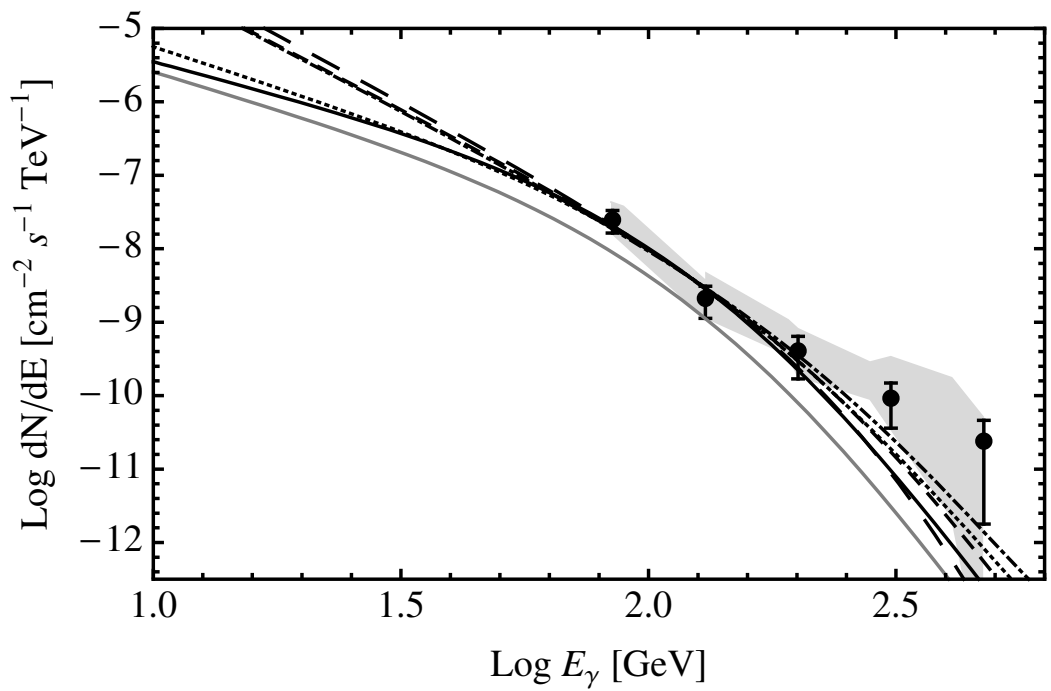

Fig. 2.- Plot of the best-fit curves with $\Gamma_{s}$ and $K$ as free parameters for the SMS fast evolution (solid), SMS baseline (dotted), Kneiske et al. best-fit (short-dashed), Kneiske et al. low-IR (longdashed), and Primack et al. (dash-dotted) models shown along with the observational data from Albert et al. (2008). The shaded region represents the combined systematic and statistical error for the observational data. The gray curve shows the EGRET flare power-law fit extended to higher energies multiplied by $e^{-\tau(E)}$. The respective $\chi^{2}$ values are given in the third number column in Table 2.

here. Assuming the value for $\Gamma_{s}$ of 2.02 , we obtain the best fits to the MAGIC data using the Levenberg-Marquardt method for the single free parameter, $K$. The $\chi^{2}$ values obtained for these best fits are shown in the last column of Table 2. The resulting curves for the fits of all five models are illustrated in figure 2 .

If we then choose the form of $\tau(E)$ given by the SMS fast evolution model as input to equation (1), by extending the $\Gamma_{s}=2.02$ power-law fit from Hartman et al. to an energy of $\sim 500 \mathrm{GeV}$, we find a value for $K$ that is $\sim 3$ times higher for the MAGIC flare than for the flare observed by EGRET 15 years earlier. Thus, we obtain a reasonable estimate for the luminosity of the MAGIC flare that is comparable to that of the earlier flare observed by EGRET.

\section{Conclusions and Discussion}

We conclude that the observational data of 3C279 from the MAGIC collaboration do not significantly constrain the intergalactic low energy photon spectra, nor do they indicate that the Universe is more transparent to high energy $\gamma$-rays than previously thought or obtained by the SMS models. Including the effects of the systematic errors, which only significantly affect the two 


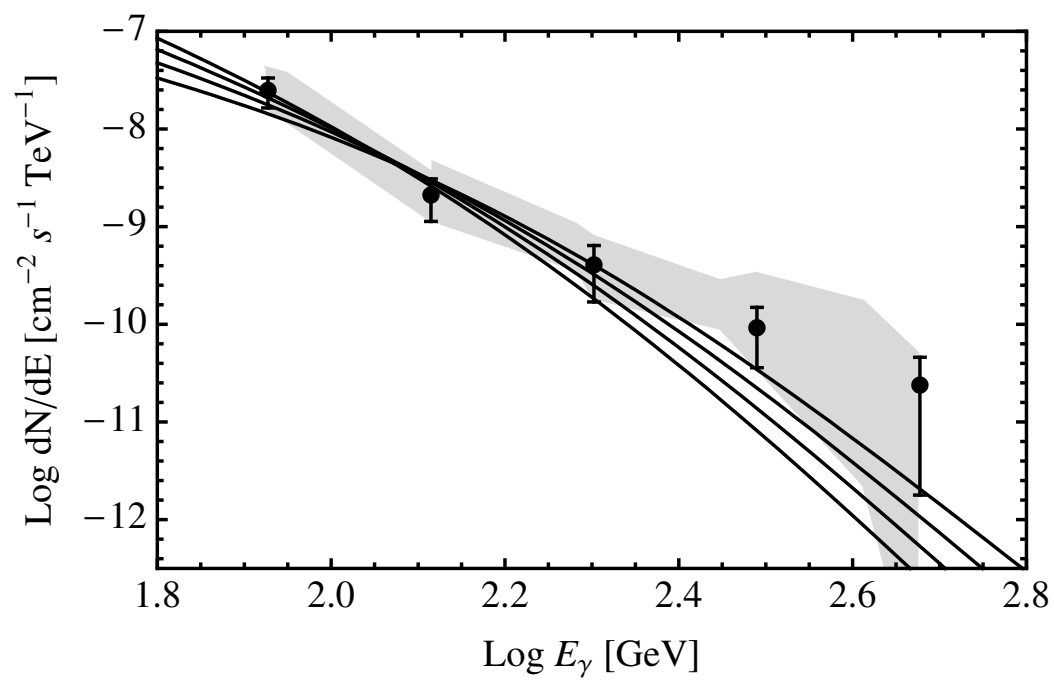

Fig. 3. - Plot of the best fit curves obtained using the SMS baseline model for fixed spectral indices of 1.5, 2.0, 2.5, and 3.0. The shaded region represents the combined systematic and statistical error for the observational data.

highest energy data points, would only strengthen our conclusions.

We show here, as well as in our analysis of 1ES0229 (Stecker \& Scully 2008), that the SMS models produce reasonable fits to the observational data. We further demonstrate that other models in the literature that give lower transparencies do not fit the $3 \mathrm{C} 279$ data better than the SMS models do. This is because the magnitude and shape of the intrinsic spectrum of the flare are not well determined by the MAGIC data, as we have shown.

The spectral energy distribution of the intergalactic IR radiation is well constrained by astronomical data, as discussed in detail in SMS. However, the form of the intrinsic source spectrum of

Table 2. Best-fit Spectral Parameters for EBL Models with $\chi^{2}$ Values

\begin{tabular}{lllll}
\hline \hline \multicolumn{1}{c}{ Model } & $K$ & $\Gamma_{s}$ & $\chi^{2}$ & $\chi^{2}$ for $\Gamma_{s}=2.02$ \\
\hline Fast Evolution & 2.15 & 1.78 & 1.59 & 1.20 \\
Baseline & 8.75 & 2.19 & 1.42 & 1.07 \\
Kneiske Low IR & 128 & 3.46 & 1.59 & 1.28 \\
Kneiske Best Fit & 283 & 3.62 & 1.55 & 1.42 \\
Primack & 141 & 3.49 & 1.54 & 1.25 \\
\hline
\end{tabular}




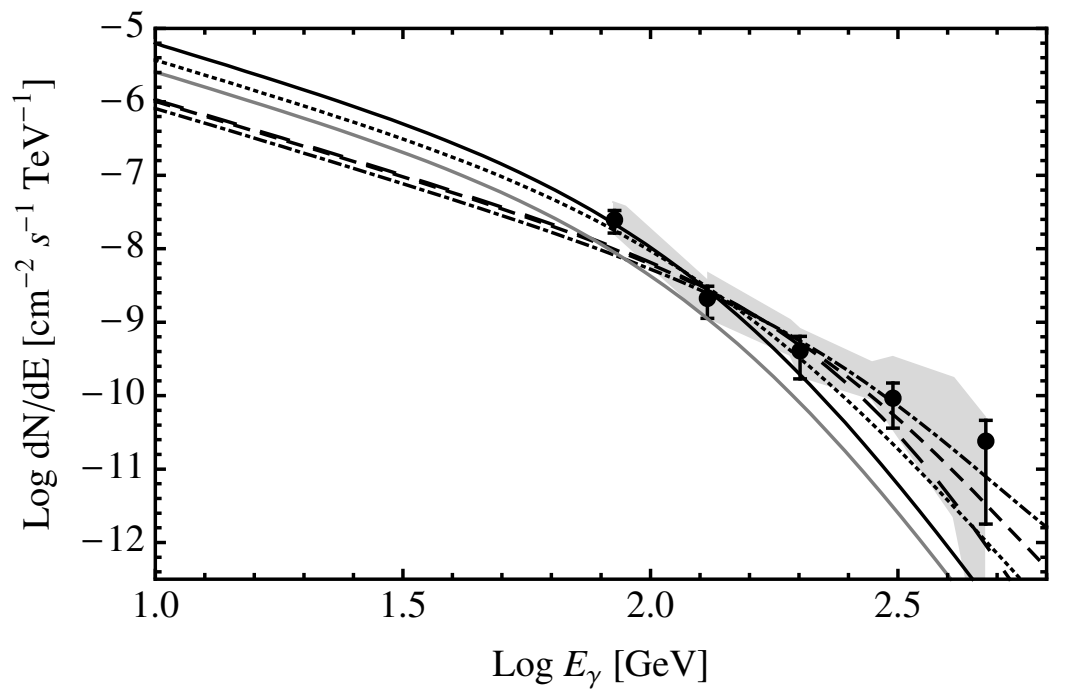

Fig. 4.- Plot of the best fit curves with $\Gamma_{s}=2.02$ for the SMS fast evolution (solid), SMS baseline (dotted), Kneiske et al. best-fit (short-dashed), Kneiske et al. low-IR (long-dashed), and Primack et al. (dash-dotted) models for a fixed spectral index of $\Gamma_{s}=2.02$ shown along with the observational data from Albert et al. (2008). The shaded region represents the combined systematic and statistical error for the observational data. The gray curve shows the EGRET flare power-law fit extended to higher energies multiplied by $e^{-\tau(E)}$. The respective $\chi^{2}$ values are given in the last column of Table 2.

the $\gamma$-ray flare is not well constrained. If one should wish to speculate on producing a fit to the MAGIC data to go through all of the points, one would require an intrinsic flare spectrum which flattens at higher energies. Such a spectrum may arise either from relativistic shock acceleration (Stecker, Baring \& Summerlin 2007; Resmi \& Bhattacharya 2008) or from intrinsic source absorption (Liu \& Bai 2006; Liu, Bai \& Ma 2008; Sitarek \& Bednarek 2008). However, given both the statistical and the systematic errors of the MAGIC data, particularly those for the highest energy bin, the invocation of such processes is unnecessary.

If we apply our analysis technique to the MAGIC data and assume an intrinsic power-law spectrum with index 2.02 as observed for the earlier EGRET flare at lower energies where absorption is negligible, we find that the luminosity of the MAGIC flare was similar to that of the earlier EGRET flare. In fact, the MAGIC flare was $\sim 3$ times brighter. This luminosity estimate could not be obtained using the analysis adopted by the MAGIC group, since all of the data points that they started from were affected by intergalactic absorption.

One would need to have additional information concerning the intrinsic source spectrum such as additional observations at lower $\gamma$-ray energies that are unaffected by pair-production absorption in order to better constrain the intergalactic IR radiation through an analysis of the resulting optical 
depth at the higher energies where absorption is more significant. We note that the ideal situation for exploring the exact amount of intergalactic absorption would be to have simultaneously and with overlapping observational energy ranges (1) observations of a strong flare with large photon counts at lower energies unaffected by intergalactic absorption, as can be obtained by FGST (Fermi Gamma-ray Space Telescope, nee GLAST), and (2) ground based sub-TeV observations of such a strong flare with larger photon counts.

We wish to thank Rudolf Bock for sending us a list of data on the spectrum of 3C279 observed by MAGIC. STS gratefully acknowledges partial support from the Thomas F. \& Kate Miller Jeffress Memorial Trust grant no. J-805.

\section{REFERENCES}

Albert, J. et al. 2008, Science 320, 1752

Altieri, B. et al. 1999, A\&A 343, L65

Breit, G. \& Wheeler, J. A. 1934, Phys. Rev. 46, 1087

Elbaz, D. et al. 2002, A\&A 384, 848

Hartman, R. C. et al. 1992, ApJ 385, L1

Hauser, M.G. \& Dwek, E. 2001 ARA\&A 39, 249

Katarzyńsky, K., Ghisellini, G., Svensson, R., Gracia, J. \& Maraschi, L. 2006, MNRAS 368, L52

Kneiske, T. M. et al. 2004, A\&A 413, 807

Lagache,G. et al. 2004, ApJS 154, 112

Liu, H. T. and Bai, J. M. 2006, ApJ 653, 1089

Liu, H. T., Bai, J. M. \& Ma, L. 2008, arXiv:0807.3133

Malkan, M. A. \& Stecker, F. W. 1998, ApJ 496, 13

Malkan, M. A. \& Stecker, F. W. 2001, ApJ 555, 641

Marziani, P. et al. 1996, ApJS 104, 37

Primack, J. R., Bullock, J. S. \& Somerville, R. S. 2005, AIP Conf. Proc. 745, 23

Resmi, L. \& Bhattacharya, D. 2008, MNRAS 388, 144

Sitarek, J \& Bednarek, W. 2008, arXiv:0807.4228 
Stecker, F. W., Baring, M.G., \& Summerlin, E.J. 2007, ApJ 667, L29

Stecker, F. W., de Jager, O. C. \& Salamon, M. H. 1992, ApJ 390, L49

Stecker, F. W., Malkan, M.A., \& Scully S.T. 2006, ApJ 648, 774

Stecker, F. W., Malkan, M. A. \& Scully, S. T. 2007, ApJ 658, 1392

Stecker, F. W., Puget, J.-L. \& Fazio, G. G. 1977, ApJ 214, L51

Stecker, F. W. \& Scully, S. T. 2006, ApJ 652, L9

Stecker, F. W. \& Scully, S. T. 2008, A\&A 478, L1

Totani, T. \& Takeuchi, T. T. 2002, ApJ 570, 470

This preprint was prepared with the AAS $\mathrm{LAT}_{\mathrm{E}} \mathrm{X}$ macros v5.0. 